\title{
Instructional Design Methodological Proposal for the Training of Online Content Tutors
}

\section{Propuesta metodológica de diseño instruccional para la formación de tutores de contenidos en-línea}

\section{Carlos Aimacaña1, Danilo Pástor², Gloria Arcos-Medina², Byron Vaca², and Alejandra Oñate ${ }^{2}$}

${ }^{1}$ Unidad Educativa san Felipe Neri

${ }^{2}$ Escuela Superior Politécnica de Chimborazo

\section{Abstract}

Corresponding Author:

Carlos Aimacaña

webmaster@mitalysoft.com

Received: 4 December 2018

Accepted: 5 December 2018

Published: 27 December 2018

Publishing services provided by Knowledge $\mathrm{E}$

(c) Carlos Aimacaña et al. This article is distributed under the terms of the Creative Commons Attribution License, which permits unrestricted use and redistribution provided that the original author and source are credited.

Selection and Peer-review under the responsibility of the SIIPRIN-CITEGC Conference Committee.
With the challenge of change that a teacher faces, the use of educational technologies requires knowledge of instructional design. The teacher plays a prominent role in this process, so it must create educational content, following the principles of an instructional design model. The problem is that not all methodologies include a practical approach where the tutor takes ownership of pedagogical and practical aspects. Therefore, this study proposes an instructional design methodology for online Tutors combining the ADDIE, ASSURE, and PACIE models. In addition, to validate the proposed methodology, an evaluation process is carried out, taking into account the appropriation of the role of tutor of online content and level of use of the LMS tools.

\section{Resumen}

Con el reto de cambio que un docente enfrenta al uso de tecnologías educativas se requiere conocimientos de diseño instruccional que apoye metodológicamente a esta transición. El docente juega un rol destacado en este proceso, por lo debe crear contenido educativo, siguiendo los principios de un modelo de diseño instruccional. El problema es que no todas las metodologías incluyen un enfoque práctico en donde el tutor se apropie de aspectos pedagogicos y practicos. Por lo tanto, este estudio propone una metodología de diseño instruccional para Tutores on-line combinando los modelos ADDIE, ASSURE y PACIE. Además, para validar la metodología propuesta se realiza un proceso de evaluación, tomando en cuenta la apropiación del rol de tutor de contenidos on-line y nivel de utilización de las herramientas LMS.

Keywords: instructional design, LMS, online course, E-learning

Palabras clave: Diseño Instruccional, LMS, Cursos en-línea, E-learning 


\section{Introducción}

El reto que enfrentan los docentes para cambiarse de la educación tradicional hacia los nuevos paradigmas de enseñanza requiere de mucho esfuerzo. Lógicamente que para éste proceso se necesita el apoyo de las nuevas tecnologías de información en el campo de la enseñanza-aprendizaje. En este sentido, los docentes generalmente intentan replicar los métodos tradicionales de enseñanza en entornos e-learning, pero en realidad este proceso implica mucho más, es decir, revisar principios y prácticas pedagógicas en donde el rol del docente es la clave principal para tener éxito [1]. Incluso existe una amplia variedad de cursos, herramientas y bibliografía que los tutores lo usan para capacitarse y así poder afrontar los procesos de enseñanza online, pero al momento de aplicar su diseño instruccional en un entorno de aprendizaje virtual lo hacen de forma superficial. Esto hace notar la creciente importancia del uso de las pedagogías a medida que aumenta la complejidad del diseño, las tecnologías y las expectativas de los usuarios en el campo del e-learning [2].

Para poder diseñar un entorno de aprendizaje se puede utilizar herramientas de diseño. Una comunmente usada es un LMS (Learning Managment System - LMS en inglés), es decir un sistema de gestión de aprendizaje, el cual es un software basado en la web que permite ejecutar cursos on-line [3]. El curso on-line (tambien Ilamado curso virtual) permite organizar los recursos de aprendizaje para simular un proceso de enseñanza-aprendizaje entre el Tutor y el estudiante, como si fuera en una clase real. Con cierto tipo de preparación los docentes podrían diseñar sus propios cursos on-line, sin embargo se percibe una mala práctica de diseño y además estos cursos se crean de manera minimalista, ya que, la mayoría de veces se tienden a reutilizar un prototipo con ligeras modificaciones [4]. Una posible causa puede ser la falta de formación de los instructores en el área del diseño instruccional. Otro motivo se debe a que existen pocas investigaciones sobre cómo los instructores diseñan cursos online [5]. En concecuencia, surge la expectativa de cómo crear cursos on-line, pero con un soporte pedagógico en diseño instruccional para que los tutores se apropien del rol docente.

De acuerdo a [6] menciona que la manera adecuada de crear contenido educativo es siguiendo los principios de un modelo de diseño instruccional. Sin embargo, existen muchas teorias y modelos que se enfocan en seguir un conjunto de pasos secuenciales o incrementales y no guian al diseñador con detalles de cómo realizar actividades [7]. Recientes estudios identifican modelos muy populares como ADDIE, ASSURE, Dick and Carey entre otros. A pesar de que la mayoría de los modelos de diseño instruccional 
se basan en el modelo ADDIE [8], existen otras alternativas muy prácticas y poco conocidas en la literatura científica como PACIE, que podrían combinarse para producir una metodología innovadora que ayude a la formación de tutores on-line.

Varios trabajos de investigación se han realizado acerca de propuestas de diseño instruccional. Uno de ellos, presenta una investigación asociada con diferentes modelos que se utilizaron para estudiar el impacto de los factores de diseño sobre la actitud, centrándose en la investigación del entorno de aprendizaje [9]. Otro trabajo relacionado formula un marco metodológico para el desarrollo de un modelo de diseño instruccional, el cual proporciona una contribución teórica y práctica [10]. En otro estudio se promueve un enfoque de mente abierta para el diseño instruccional, utiliza una gama de técnicas para abordar una amplia variedad de tareas de diseño instruccional, tanto en el contexto de la educación formal como formación profesional e industrial [11]. En otro contexto, un estudio desarrolló un modelo de diseño de "Flipped Learning" que apoyaba sistemáticamente a los instructores en la creación de conferencias on-line [12]. Todos los trabajos descritos anteriormente siguen un lineamiento de aplicación sistemática de un modelo de diseño instruccional, pero no consideran aspectos prácticos que ayuden a crear el contenido a los tutores en una herramienta determinada.

Una vez realizada la revisión bibliográfica se concluye que no todos los modelos de diseño instruccional, incluyen un enfoque práctico en donde el tutor se apropie de aspectos pedagógicos y prácticos para facilitar la creación de contenido educativo. El objetivo principal del presente trabajo es proponer una metodología de diseño instruccional para Tutores on-line combinando los modelos ADDIE, ASSURE y PACIE. Además, para validar la metodología propuesta se realiza un proceso de evaluación, tomando en cuenta la apropiación del rol de tutor de contenidos on-line y nivel de utilización de las herramientas LMS.

El paper está organizado de la siguiente manera: la sección 2 describe el marco teórico que ayuda a fundamentar el presente trabajo; la sección 3 especifica la propuesta metodologica de diseño instruccional; la sección 4 detalla la metodología de investigación para validar la propuesta; la sección 5 realiza un análisis de los resultados obtenidos de la evaluación ejecutada a un equipo de docentes, y finalmente la sección 6 muestra las conclusiones del presente trabajo y los posibles trabajos futuros.

\section{Marco teórico}




\subsection{Diseño Instruccional}

El concepto de Diseño Instruccional fue introducido por Robert Glaser en 1961, su concepción se fundamenta en la tecnología educativa, que ha sido entendida como la aplicación de la tecnología para la elaboración de recursos de aprendizaje desde el diseño hasta la utilización de estos; su objetivo es mejorar el desempeño profesional de los estudiantes a partir de la adquisición de competencias [13].

El diseño instruccional supone una planificación instruccional sistemática que incluye la valoración de necesidades, el desarrollo, la evaluación, la implementación y el mantenimiento de materiales y programas [14], y debe asumirse como un andamiaje que permita integrar los avances de las TIC's y las innovaciones y los retos del proceso de enseñanza y de aprendizaje [15]. Además, ha sido concebido como la planificación de la educación que implica la elaboración de guiones, planes, proyectos, y que generalmente se lleva a cabo bajo procedimientos estandarizados [16].

Las diferentes concepciones del diseño instruccional son expresadas a través de los Modelos de Diseño Instruccional que sirven de guía a los profesionales sistematizando el proceso de desarrollo de acciones formativas [17], su objetivo es orientar hacia el diseño y presentación de contenidos educativos y sus correspondientes actividades de aprendizaje y evaluación, y se agrupan en modelos de primera y segunda generación: a) Modelos de primera generación: orientados a la tecnología educativa y procesos genéricos; entre ellos se encuentran el modelo ADDIE, ASSURE, modelo de Dick, el de Davis entre otros, b) Modelos segunda generación, orientados hacia los conceptos de diseño de aprendizaje o teorías pedagógicas entre ellos ARC, Gagné y Briggs, Elaboration Theory y otros [13].

\subsection{Modelo ADDIE}

El modelo ADDIE es un modelo que se caracteriza por englobar las principales fases del diseño instruccional [18], su nombre obedece al acrónimo analize (análisis), design (diseño), develop (desarrollo), implement (implementación) y evaluate (evaluación); que representan las fases de este modelo. Este modelo representa un punto de construcción para otros modelos de diseño instruccional, porque "es un marco de trabajo general" [19], además, es considerado un modelo que es válido para cualquier contexto educativo [20]. Las fases del modelo son las siguientes:

Análisis: Evalúa las necesidades del entorno, define el problema, identifica qué causa el problema y buscar posibles soluciones. 
Diseño: Determina cómo alcanzar las metas educativas obtenidas durante la fase de análisis.

Desarrollo: Genera las unidades, módulos y los materiales didácticos.

Implementación: Pone en práctica el programa instruccional de forma eficiente y efectiva.

Evaluación: Realiza una evaluación formativa (a lo largo de todo el proceso) y también sumativa (al final del proceso de formación).

El modelo ADDIE es un proceso iterativo de diseño instruccional, donde los resultados de la evaluación formativa de cada fase puede llevar al diseñador instruccional a regresar a cualquier fase previa [21].

\subsection{Modelo ASSURE}

Heinich, Molenda, Russell y Smaldino (1993) desarrollaron el modelo ASSURE, este representa una guía para planear y conducir la enseñanza aprendizaje apoyado en las Tecnologías de Información y Comunicación [22]. De acuerdo a [23] con el acrónimo ASSURE se abrevian 6 fases que componen el modelo:

A - analizar a los aprendices (Analyze learners),

$\mathrm{S}$ - formular los objetivos de aprendizaje (State learning objectives),

$\mathrm{S}$ - seleccionar métodos, medios y materiales (Select methods, media and materials),

$\mathrm{U}$ - utilizar métodos, medios y materiales (Utilize methods, media and material),

$\mathrm{R}$ - requerir la participación de los aprendices (Require learner participation), y

E - evaluar (Evaluate and revise).

\subsection{Metodología PACIE}

La metodología PACIE es una metodología para el uso y aplicación de herramientas virtuales en la educación [24], esta metodología permite desarrollar en el estudiante un aprendizaje colaborativo mediante la interacción y desenvolvimiento en un LMS, además de aportar beneficios a nivel organizacional, académico, pedagógico, comunicacional, y tecnológico [25]. 
Es una metodología que permite el uso de recursos de tipo cognitivo y motiva la construcción de conocimiento a través de actividades de tipo interactivo y comunicacional [24]. A pesar de que no está tan difundida en la comunidad científica, ha sido divulgada a través de muchos programas de capacitación en toda Latinoamérica [4].

El nombre PACIE es el resultado de las iniciales de cada uno de los procesos secuenciales en los que se fundamenta esta metodología:

P- presencia: se debe agotar todos los mecanismos posibles para que los alumnos ingresen y participen en las aulas virtuales y que se sientan motivados, adquiriendo y compartiendo conocimiento.

A-alcance: Se debe tener objetivos claros, que señalen realmente aquello que queremos conseguir de los estudiantes, que determinen claramente los objetivos que se debe alcanzar.

C- capacitación: El Tutor debe estar debidamente preparado para emprender el reto, capacitado, seguro, pronto para poder auxiliar a los educandos cuando estos lo requieran.

I-interacción: Los recursos y actividades deben ser utilizados para socializar y compartir, para generar interacción, para estimular, y para guiar y acompañar.

E-elearning: Usar toda la tecnología a nuestro alcance pero sin olvidar la Pedagogía, que es la guía fundamental de todo el proceso educativo y que el docente debe conocer muy bien, enseñar, crear apoyándose en la red [24].

\section{Propuesta Metodológica de Diseño Instruccional}

\subsection{Aspectos Generales}

La propuesta metodológica planteada en la presente investigación lleva en sí el propósito de delinear los argumentos necesarios para su utilización en el correcto manejo de los Learning Management Systems tanto metodológicamente como al nivel de la propia plataforma - herramienta desde la perspectiva del educador virtual y así cumplir con su objetivo fundamental de aportar con una Propuesta Metodológica para la utilización de los "Learning Management Systems" enfocada a la formación de Tutores de Contenidos Online.

El proceso metodológico que debe aplicar un tutor en línea, se desarrollará adoptando características relevantes de los lineamientos de los modelos instruccionales 
teniendo como base principal las metodologías ADDIE, ASSURE y PACIE, como se muestra en la Figura 1, la metodología propuesta, representada en el centro, considera las características de las metodologías indicadas.

La propuesta apoya al adecuado desempeño del Tutor, ya que posee las siguientes características:

- Analiza la situación y características del estudiante participante.

- Facilita la introducción progresiva al e-learning de manera lineal.

- Colabora al nivel del Tutor/Docente para el aprovechamiento de la tecnología a favor del estudiante.

- Crea un ámbito no solo procedimental sino además un ambiente con calidad y calidez.

- Evita duplicar los procesos administrativos y educativos y su interrelación.

- Crea un sentido de pertenencia personal e institucional en el ambiente del LMS

- Jerarquiza la información de los contenidos, recursos y actividades.

- Propende generar un entorno cómodo y amigable en el concepto del LMS de la mano de mantener una imagen formal y/o corporativa.

- Planifica una secuencia de inmersión haciendo un correcto proceso de inducción y minimizando rechazo.

- Utiliza el paradigma constructivista.

- Propone un ciclo de desarrollo análogo a las recomendaciones generales de ADDIE para mantener a nivel de proceso el análisis, diseño, desarrollo, implementación y su continua evaluación.

- Genera procesos interactivos en el aula así como procesos comunicacionales de apoyo y comunidad.

- Evoluciona a partir del avance de las TICs.

- Propende a una adecuada definición de las técnicas de evaluación y autoevaluación.

\subsection{Fases del Modelo Propuesto}

La propuesta metodológica consta de seis fases, las mismas que se resumen la la Tabla 1. 


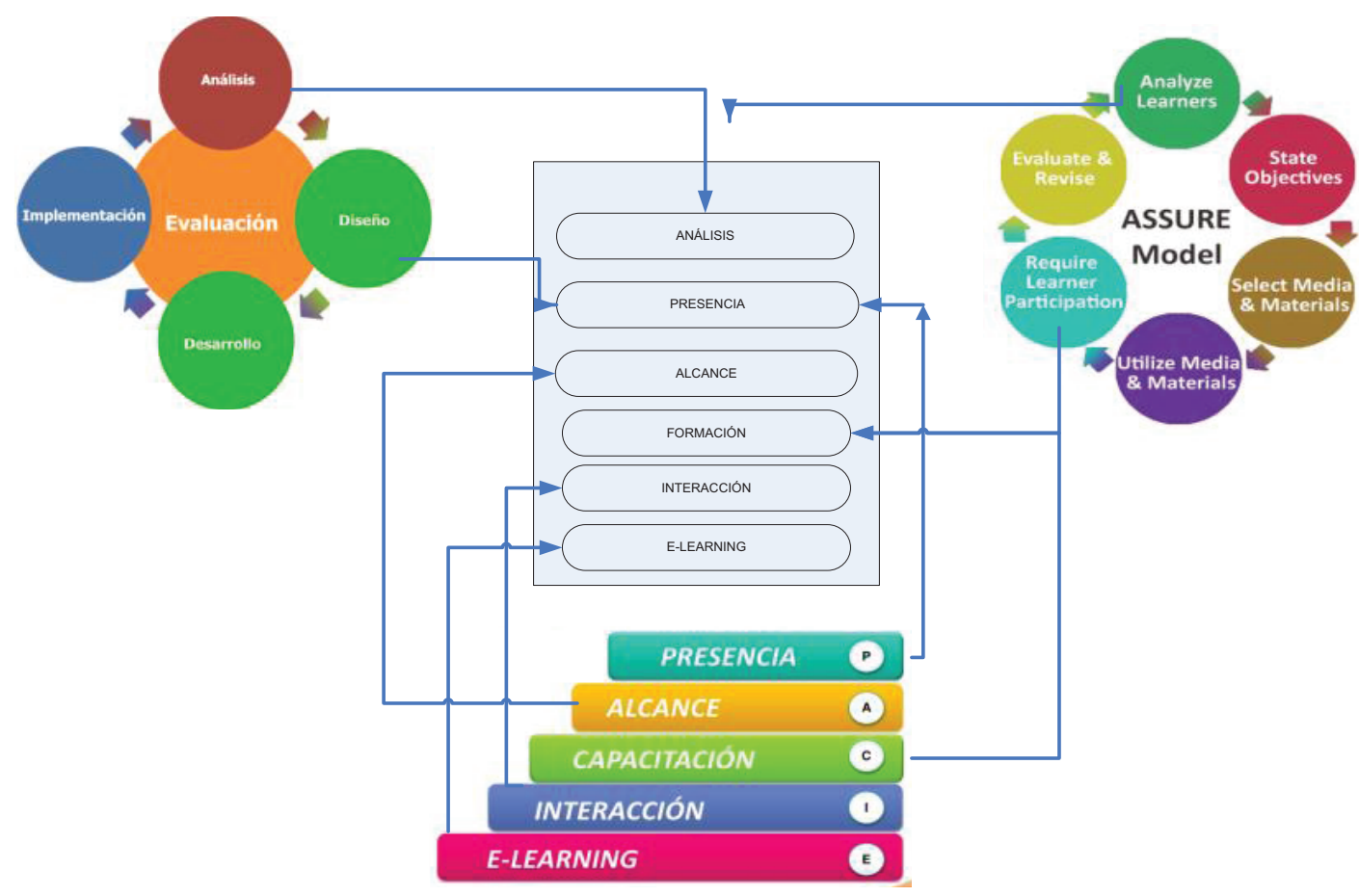

Figura 1: Propuesta Metodológica y su relación con otras metodologías.

TABLA 1: Fases de la propuesta metodológica.

Fase
Análisis del
participante
Presencia
Alcance
Formación /
Capacitación
Interacción
E-learning

\section{Descripción}

Identificación del estudiante en función del nivel que cursa, su edad, antecedentes escolares, intereses, relación con la tecnología, género, etc.

Definición de la imagen corporativa, arquitectura de la información y parámetros de usabilidad.

Organización de la información en base a los objetivos del curso.

El tutor adoptará su rol en las etapas del ciclo del diseño, conjuntamente con las actividades de investigar, planificar, crear, evaluar, con autonomía como compromiso dentro del proceso.

Establecimiento del nivel de interacción, nivel de comunicación, niveles de estimulación y motivación.

Verificación de los avances y resultados del proceso en función de sus actores, así como de la retroalimentación ligando las etapas anteriores para una mejora continua.

\subsection{Roles del Tutor de Contenidos Online}

En correspondencia con la estructura de la metodología, se propone además los roles que un Tutor On-line debe desempeñar durante el proceso de enseñanza, que se presentan en la Tabla 2. 
TABLA 2: Roles del Tutor online.

Rol
Área pedagógica
Área social
Área administrativa
Facilitador
organizacional dentro
del aula virtual
Facilitador intelectual
dentro del aula virtual
Área técnica

\section{Implicación}

Objetivos claros, gestión comunicacional, promover la investigación, seleccionar y poner a disposición los contenidos para la enseñanza.

Romper el hielo, aplaudir y moderar, promover la comunicación.

Informar tiempos y alcances, distribución de participantes, establecimiento de roles.

Estimular y motivar la participación, organizar los grupos de trabajo, enrumbar los criterios, organización de jerarquías de información.

Objetividad, promover la investigación y la generación de conocimientos, resolver dudas, ampliar los conocimientos.

Soporte de primera mano, puente entre el área técnica, ajuste de tiempos según se requiera.

\section{Metodología de la Investigación}

Para cumplir con el objetivo de la investigación, se consideró una muestra de 12 docentes, de una unidad educativa reconocida de la ciudad de Riobamba, Ecuador; quienes tenían conocimientos previos sobre la utilización de LMS y su aplicación en el proceso de enseñanza y aprendizaje.

El diseño de la investigación fue cuasi-experimental, ya que se recopilaron datos para comparar las mediciones de comportamiento de un grupo control, con las mediciones de un grupo experimental. Los escenarios aplicados fueron los siguientes: Escenario 1(Grupo de control), sin la utilización de la metodología propuesta y Escenario 2 (Grupo experimental), luego de un proceso de formación aplicando la metodología propuesta. En el Escenario 2, cada docente implementó un aula virtual sobre la plataforma MOODLE, de acuerdo a lo aprendido en el proceso de formación.

Las variables analizadas en los dos escenarios fueron: Nivel de apropiación del rol de tutor de contenidos On-line y el Nivel de utilización de las herramientas del LMS. Los indicadores de las variables se muestran en la Tabla 3.

Los datos fueron recopilados a través de la técnica de la encuesta, la misma que fue aplicadas en cada escenario, Los cuestionarios aplicados constaron de 12 preguntas: seis preguntas correspondientes a los indicadores de la variable Nivel de apropiación del rol de tutor de contenidos On-line y seis relacionadas con los indicadores de la variable Nivel de utilización de las herramientas del LMS. Las preguntas fueron evaluadas aplicando una escala de Likert del 1 al 5 donde, 1=Nunca, 2=Rara vez, 3=Algunas veces, 4=Casi siempre, 5=Siempre.

Las hipótesis de investigación planteada en este estudio, es la siguiente: 
Hi: El Nivel de apropiación del rol de tutor de contenidos On-line y el Nivel utilización de las herramientas del LMS en el Aula Virtual son dependientes de la aplicación de la metodología usada para la formación de tutores On-line propuesta.

Ho: El Nivel de apropiación del rol de tutor de contenidos On-line y el Nivel utilización de las herramientas del LMS en el Aula Virtual son independientes de la aplicación de la metodología usada para la formación de tutores On-line propuesta.

TABLA 3: Variables e indicadores del estudio.

\begin{tabular}{|c|c|c|}
\hline Variable Dependiente & Id & Indicador \\
\hline \multirow[t]{6}{*}{$\begin{array}{l}\text { V1. Nivel de apropiación del rol } \\
\text { de tutor de contenidos On-line }\end{array}$} & $\mid 1.1$ & $\begin{array}{l}\text { Aplicación de los criterios componentes del manejo } \\
\text { del área pedagógica - Objetivos claros, gestión } \\
\text { comunicacional, promover la investigación }\end{array}$ \\
\hline & 1.2 & $\begin{array}{l}\text { Aplicación de los criterios componentes del manejo } \\
\text { del área social - romper el hielo, aplaudir y moderar }\end{array}$ \\
\hline & $\mid 1.3$ & $\begin{array}{l}\text { Conocimiento de los lineamientos del manejo del área } \\
\text { administrativa - Informar tiempos y alcances, } \\
\text { distribución de participantes }\end{array}$ \\
\hline & 11.4 & $\begin{array}{l}\text { Cumplimiento del rol de Facilitador Organizacional } \\
\text { dentro del Aula Virtual - Estimular y motivar la } \\
\text { participación, organizar los grupos de trabajo, } \\
\text { enrumbar los criterios }\end{array}$ \\
\hline & 11.5 & $\begin{array}{l}\text { Cumplimiento del rol de Facilitador Intelectual dentro } \\
\text { del Aula Virtual - Objetividad, promover la } \\
\text { investigación y la generación de conocimientos, } \\
\text { resolver dudas, ampliar los conocimientos }\end{array}$ \\
\hline & 11.6 & $\begin{array}{l}\text { Cumplimiento del rol en el área técnica - Soporte de } \\
\text { primera mano, puente entre el área técnica, ajuste de } \\
\text { tiempos según se requiera. }\end{array}$ \\
\hline \multicolumn{3}{|l|}{$\begin{array}{l}\text { V2. Nivel de utilización de las } \\
\text { herramientas LMS }\end{array}$} \\
\hline \multirow[t]{2}{*}{$\begin{array}{l}\text { P1. Nivel de Personalización de } \\
\text { la Interfaz del LMS. }\end{array}$} & $\mid 2.1$ & $\begin{array}{l}\text { Utilización de las herramientas para la maquetación } \\
\text { del Entorno Virtual de Aprendizaje. }\end{array}$ \\
\hline & 12.2 & $\begin{array}{l}\text { Envío de tareas a través de la opción en actividades } \\
\text { de "Tarea". }\end{array}$ \\
\hline $\begin{array}{l}\text { P2. Nivel de conocimiento de los } \\
\text { entornos de comunicación. }\end{array}$ & 12.3 & $\begin{array}{l}\text { Manejo de la herramienta de la Actividad "Foro de } \\
\text { Discusión". }\end{array}$ \\
\hline $\begin{array}{l}\text { P3. Nivel de Identificación y } \\
\text { conocimiento de las } \\
\text { Herramientas de diagramación } \\
\text { de contenidos }\end{array}$ & 12.4 & Utilización de la herramienta de contenidos "Libro". \\
\hline \multirow[t]{2}{*}{$\begin{array}{l}\text { P4. Nivel de desempeño usando } \\
\text { las herramientas de Evaluación }\end{array}$} & 12.5 & $\begin{array}{l}\text { Utilización en el LMS de la herramienta de bitácora } \\
\text { "Diario". }\end{array}$ \\
\hline & 12.6 & $\begin{array}{l}\text { Utilización de la herramienta de Evaluación } \\
\text { "Cuestionario". }\end{array}$ \\
\hline
\end{tabular}

Como se puede ver en la Tabla 3, los indicadores de la variable Nivel de apropiación del rol de tutor de contenidos On-line, corresponden a los roles del tutor On-line de la metodología propuesta. Por otro lado, los indicadores de la variable Nivel utilización de 
las herramientas del LMS en el Aula Virtual, corresponden a la aplicación de actividades y recursos del LMS.

Para la prueba de hipótesis se utilizó Chi-cuadrado para demostrar la asociación entre las variables, y luego para evaluar en qué medida se ajusta la distribución de frecuencias obtenida con los datos, a una distribución teórica o esperada contrastando frecuencias observadas con las frecuencias esperadas de acuerdo con la hipótesis nula. Se utilizó un nivel de significación alfa=0,05, es decir que si el valor de Chi-cuadrado calculado para un experimento es mayor que el correspondiente al de la probabilidad del $5 \%$ se rechaza la hipótesis.

\section{Resultados}

Los resultados obtenidos de las encuestas, tanto en el grupo de control como en el grupo experimental, sirvieron de base para generar las tablas de consistencia de cada variable de estudio. El nivel de cada variable ha sido considerado como Alto, para los valores 4 y 5 de la escala de Likert, Medio para el valor 3 y Bajo para los valores 1 y 2 de dicha escala.

El número de observaciones realizadas es 72, para cada escenario de prueba, obtenidos del análisis de seis indicadores correspondientes a los 12 docentes que formaron parte de la muestra; dando un total de 144 observaciones para los dos escenarios.

\subsection{Análisis de la Variable 1 (V1): Nivel de apropiación del rol de tutor de contenidos On-line}

La Tabla 4, presenta los resultados obtenidos de cada indicador de la variable Nivel de apropiación del rol de tutor de contenidos On-line, para los escenarios de control y experimentación. Los resultados muestran un incremento en el nivel Alto y Medio del $80,5 \%$ y $9,7 \%$ respectivamente y una disminución del nivel Bajo en el 90,3\%; esto evidencia la que la metodología instruccional propuesta contribuye en la mejora del nivel de apropiación del rol del tutor. Las mejoras más significativas corresponden a los indicadores Área Administrativa y Área Técnica, con el 91,7\% y 83,4\% respectivamente, en lo que se refiere al nivel alto de evaluación de esta variable; es decir, que los tutores adquirieron la habilidad de informar tiempos y alcances, distribuir los participantes de acuerdo a las actividades planificadas; así como, realizar cambios de configuración del aula virtual que son responsabilidad del tutor On-line. 
La Tabla 5 presenta los resultados de la prueba estadística Chi cuadrado para la primera variable de estudio, de acuerdo a esta prueba se considera que:

$\mathrm{P}$ valor (significación asintótica) $<0,05$ Se rechaza la Ho y se acepta la Hi

$\mathrm{P}$ valor (significación asintótica) > 0,05 Se acepta la Ho y se rechaza la Hi

Los resultados presentan un $\mathrm{P}$ valor $=0,000$, que es menor que 0,05 , por lo tanto se rechaza la Ho y se acepta la Hi, es decir, que El Nivel de apropiación del rol de tutor de contenidos On-line y el Nivel utilización de las herramientas del LMS en el Aula Virtual son dependientes de la aplicación de la metodología usada para la formación de tutores On-line propuesta. Los resultados evidencian que el nivel de apropiación del rol tutor de contenidos es mejor cuando se utiliza la metodología propuesta.

TABLA 4: Tabla de Contingencia - Variable: Nivel de apropiación del tol de tutor de contenido On-Line.

\begin{tabular}{|c|c|c|c|c|c|}
\hline \multirow[t]{2}{*}{ Grupo } & \multirow[t]{2}{*}{ Indicadores } & \multicolumn{3}{|c|}{$\begin{array}{l}\text { Nivel de apropiación del rol de tutor de } \\
\text { contenidos On-line }\end{array}$} & \multirow[t]{2}{*}{ Total } \\
\hline & & Alto & Bajo & Medio & \\
\hline \multirow{14}{*}{ Control } & \multirow{2}{*}{ I1.3 A. Administrativa } & 0 & 11 & 1 & 12 \\
\hline & & $0,0 \%$ & $91,7 \%$ & $8,3 \%$ & $100,0 \%$ \\
\hline & \multirow{2}{*}{ I1.5 A. Intelectual } & 0 & 12 & 0 & 12 \\
\hline & & $0,0 \%$ & $100,0 \%$ & $0,0 \%$ & $100,0 \%$ \\
\hline & \multirow{2}{*}{ I1.4 A. Organizativa } & 0 & 11 & 1 & 12 \\
\hline & & $0,0 \%$ & $91,7 \%$ & $8,3 \%$ & $100,0 \%$ \\
\hline & \multirow{2}{*}{ I1.1 A. Pedagógica } & 1 & 10 & 1 & 12 \\
\hline & & $8,3 \%$ & $83,3 \%$ & $8,3 \%$ & $100,0 \%$ \\
\hline & \multirow{2}{*}{ I1.2 A. Social } & 0 & 12 & 0 & 12 \\
\hline & & $0,0 \%$ & $100,0 \%$ & $0,0 \%$ & $100,0 \%$ \\
\hline & \multirow{2}{*}{ I1.6 A. Técnica } & 1 & 11 & 0 & 12 \\
\hline & & $8,3 \%$ & $91,7 \%$ & $0,0 \%$ & $100,0 \%$ \\
\hline & \multirow{2}{*}{ Total } & 2 & 67 & 3 & 72 \\
\hline & & $2,8 \%$ & $93,1 \%$ & $4,2 \%$ & $100,0 \%$ \\
\hline \multirow{14}{*}{ Experimental } & \multirow{2}{*}{ I1.3 A. Administrativa } & 11 & 0 & 1 & 12 \\
\hline & & $91,7 \%$ & $0,0 \%$ & $8,3 \%$ & $100,0 \%$ \\
\hline & \multirow{2}{*}{ I1.5 A. Intelectual } & 10 & 1 & 1 & 12 \\
\hline & & $83,3 \%$ & $8,3 \%$ & $8,3 \%$ & $100,0 \%$ \\
\hline & \multirow{2}{*}{ I1.4 A. Organizativa } & 9 & 0 & 3 & 12 \\
\hline & & $75,0 \%$ & $0,0 \%$ & $25,0 \%$ & $100,0 \%$ \\
\hline & \multirow{2}{*}{ I1.1 A. Pedagógica } & 10 & 0 & 2 & 12 \\
\hline & & $83,3 \%$ & $0,0 \%$ & $16,7 \%$ & $100,0 \%$ \\
\hline & \multirow{2}{*}{ I1.2 A. Social } & 9 & 0 & 3 & 12 \\
\hline & & $75,0 \%$ & $0,0 \%$ & $25,0 \%$ & $100,0 \%$ \\
\hline & \multirow{2}{*}{ I1.6 A. Técnica } & 11 & 1 & 0 & 12 \\
\hline & & $91,7 \%$ & $8,3 \%$ & $0,0 \%$ & $100,0 \%$ \\
\hline & \multirow{2}{*}{ Total } & 60 & 2 & 10 & 72 \\
\hline & & $83,3 \%$ & $2,8 \%$ & $13,9 \%$ & $100,0 \%$ \\
\hline
\end{tabular}


TABla 5: Prueba de Chi-cuadrado -Variable: Nivel de apropiación del rol de tutor de contenido on-line.

\begin{tabular}{|l|l|c|c|c|}
\hline Grupo & Valor & gl & $\begin{array}{c}\text { Sig. } \\
\text { asintótica (2 } \\
\text { caras) }\end{array}$ \\
\hline Control & Chi-cuadrado de Pearson & 7,254 & 10 & 0,000 \\
\hline Experimental & N de casos válidos & 72 & & \\
\hline & Chi-cuadrado de Pearson & 8,800 & 10 & 0,000 \\
\hline Total & N de casos válidos & 72 & & \\
\hline & Chi-cuadrado de Pearson & 6,188 & 10 & 0,000 \\
\hline
\end{tabular}

\subsection{Análisis de la Variable 2 (V2): Nivel de utilización de las herramientas LMS}

En la Tabla 6 se presentan los resultados obtenidos de cada indicador de la variable Nivel de utilización de las herramientas $L M S$, para los escenarios de control y experimentación. Los resultados muestran un incremento en el nivel Alto y Medio del $68,15 \%$ y $2,78 \%$ respectivamente y una disminución del nivel Bajo en el $65,28 \%$; esto evidencia la que la metodología de diseño instruccional propuesta contribuye en la mejora del nivel de utilización de los recursos y actividades que proporciona el LMS. Las mejoras más significativas corresponden a los indicadores de Maquetación y Tareas, en un $100 \%$ y $83,3 \%$ respectivamente, en lo que se refiere al nivel alto de evaluación de esta variable; es decir, que los tutores mejoraron en el nivel de personalización de la interfaz y en la configuración de tareas, que es una de las actividades básicas que realizan los estudiantes. Los indicadores Cuestionario y Diario, son los que mejoraron en menor porcentaje, $66,7 \%$ y $41,7 \%$ respectivamente; una causa, puede ser, la falta de formación de los tutores en el diseño de este tipo de instrumentos de evaluación del aprendizaje para que sean aplicados en el aula virtual.

En la Tabla 7 se presentan los resultados de la prueba estadística Chi cuadrado para la segunda variable de estudio. Los resultados presentan un $\mathrm{P}$ valor $=0,000$, que es menor que 0,05, por lo tanto se rechaza la $\mathrm{Ho}$ y se acepta la $\mathrm{Hi}$, es decir, que el Nivel utilización de las herramientos del LMS en el Aula Virtual son dependientes de la aplicación de la metodología usada para la formación de tutores On-line propuesta. Los resultados evidencian que el nivel utilización de las herramientas del LMS es mejor cuando se utiliza la metodología propuesta. 
TABLA 6: Tabla de Contingencia de la Variable: Nivel de utilización de las herramientas LMS.

\begin{tabular}{|c|c|c|c|c|c|}
\hline \multirow{2}{*}{ Grupo } & \multirow[b]{2}{*}{ Indicadores } & \multicolumn{3}{|c|}{ NIVEL_V2 } & \multirow[b]{2}{*}{ Total } \\
\hline & & Alto & Bajo & Medio & \\
\hline \multirow[t]{14}{*}{ CONTROL } & \multirow[t]{2}{*}{ I2.6 Cuestionario } & 0 & 9 & 3 & 12 \\
\hline & & $0.0 \%$ & $75.0 \%$ & $25.0 \%$ & $100.0 \%$ \\
\hline & \multirow[t]{2}{*}{ I2.5 Diario } & 1 & 11 & 0 & 12 \\
\hline & & $8.3 \%$ & $91.7 \%$ & $0.0 \%$ & $100.0 \%$ \\
\hline & \multirow[t]{2}{*}{ I2.3 Foro } & 0 & 9 & 3 & 12 \\
\hline & & $0.0 \%$ & $75.0 \%$ & $25.0 \%$ & $100.0 \%$ \\
\hline & \multirow[t]{2}{*}{ I2.4 Libro } & 0 & 11 & 1 & 12 \\
\hline & & $0.0 \%$ & $91.7 \%$ & $8.3 \%$ & $100.0 \%$ \\
\hline & \multirow[t]{2}{*}{ I2.1 Maquetación } & 0 & 9 & 3 & 12 \\
\hline & & $0.0 \%$ & $75.0 \%$ & $25.0 \%$ & $100.0 \%$ \\
\hline & \multirow[t]{2}{*}{ I2.2 Tarea } & 2 & 3 & 7 & 12 \\
\hline & & $16.7 \%$ & $25.0 \%$ & $58.3 \%$ & $100.0 \%$ \\
\hline & \multirow[t]{2}{*}{ Total } & 3 & 52 & 17 & 72 \\
\hline & & $4.2 \%$ & $72.2 \%$ & $23.6 \%$ & $100.0 \%$ \\
\hline \multirow[t]{14}{*}{ EXPERIMENTAL } & \multirow[t]{2}{*}{ I2.6 Cuestionario } & 8 & 1 & 3 & 12 \\
\hline & & $66.7 \%$ & $8.3 \%$ & $25.0 \%$ & $100.0 \%$ \\
\hline & \multirow[t]{2}{*}{ I2.5 Diario } & 6 & 2 & 4 & 12 \\
\hline & & $50.0 \%$ & $16.7 \%$ & $33.3 \%$ & $100.0 \%$ \\
\hline & \multirow[t]{2}{*}{ I2.3 Foro } & 10 & 0 & 2 & 12 \\
\hline & & $83.3 \%$ & $0.0 \%$ & $16.7 \%$ & $100.0 \%$ \\
\hline & \multirow[t]{2}{*}{ I2.4 Libro } & 4 & 2 & 6 & 12 \\
\hline & & $33.3 \%$ & $16.7 \%$ & $50.0 \%$ & $100.0 \%$ \\
\hline & \multirow[t]{2}{*}{ I2.1 Maquetación } & 12 & 0 & 0 & 12 \\
\hline & & $100.0 \%$ & $0.0 \%$ & $0.0 \%$ & $100.0 \%$ \\
\hline & \multirow[t]{2}{*}{ I2.2 Tarea } & 12 & 0 & 0 & 12 \\
\hline & & $100.0 \%$ & $0.0 \%$ & $0.0 \%$ & $100.0 \%$ \\
\hline & \multirow[t]{2}{*}{ Total } & 52 & 5 & 15 & 72 \\
\hline & & $72.2 \%$ & $6.9 \%$ & $20.8 \%$ & $100.0 \%$ \\
\hline
\end{tabular}

TABLA 7: Prueba de Chi-Cuadrado - Variable: Nivel de utilización de las herramientas LMS.

\begin{tabular}{|l|l|c|c|c|}
\hline Grupo & Valor & gl & $\begin{array}{c}\text { Sig. } \\
\text { asintótica }\end{array}$ \\
\hline Control & Chi-cuadrado de Pearson & 22,176 & 10 & 0,000 \\
\hline Experimental & N de casos válidos & 72 & & \\
\cline { 2 - 4 } & Chi-cuadrado de Pearson & 22,954 & 10 & 0,000 \\
\hline Total & N de casos válidos & 72 & & \\
\hline & Chi-cuadrado de Pearson & 16,678 & 10 & 0,000 \\
\hline & N de casos válidos & 144 & & \\
\hline
\end{tabular}

\section{Conclusiones}

Esta investigación propone una Metodología de Diseño Instruccional para Tutores Online, la misma que está compuesta de seis fases: Análisis, Presencia, Alcance, Formación, Interacción y E-learning; el alcance de estas fases se basa en los modelos ADDIE, ASSURE y PACIE, que fueron analizados para realizar esta propuesta. Un aspecto importante de esta metodología es la identificación de seis roles que un tutor on-line 
debe cumplir dentro del proceso de enseñanza y aprendizaje utilizando LMS; estos roles están relacionados con los aspectos: pedagógico, social, administrativo, técnico, facilitador organizacional y facilitador intelectual dentro del aula virtual.

La metodología propuesta fue aplicada en la formación de 12 tutores on-line, los datos recopilados permitieron analizar dos variables: Nivel de apropiación del rol de tutor de contenidos On-line y Nivel de utilización de las herramientas LMS, obteniéndose como resultado una mejora en cada una de ellas del 80,5\% y 68,15\% respectivamente. La prueba estadística de Chi-cuadrado aplicada determinó una dependencia entre estas variables y la metodología de diseño instruccional propuesta.

El presente estudio puede ampliar su ámbito de investigación, ya que, la propuesta fue creada a partir del análisis de tres metodologías, sin embargo, se podría considerar aumentar otras modelos formales o informales para producir una nueva metodología con varias aristas instruccionales. También se podría combinar la metodología de diseño instruccional propuesta con alguna teoría de diseño instruccional específica.

\section{Referencias}

[1] Rodriguez, M.E., Serra, M., Cabot, J., Guitart, I.: Evolution of the Teacher Roles and Figures in E-learning Environments. In: Sixth IEEE International Conference on Advanced Learning Technologies (ICALT'06). Pp. 512-514. IEEE (2006).

[2] Haq, A. ul, Magoulas, G., Jamal, A., Majeed, A., Sloan, D.: Users' perceptions of elearning environments and services effectiveness: The emergence of the concept functionality model. J. Enterp. Inf. Manag. 31, 89-111 (2017).

[3] Fry, H., Ketteridge, S., Marshall, S.: A handbook for teaching and learning in higher education: enhancing academic practice. Routledge (2008).

[4] Pástor, D., Jiménez, J., Arcos, G., Romero, M., Urquizo, L.: Patrones de diseño para la construcción de cursos on-line en un entorno virtual de aprendizaje. Ingeniare Rev. Chil. Ing. 26, 157-171 (2018).

[5] Baldwin, S., Ching, Y.-H., Friesen, N.: Online Course Design and Development Among College and University Instructors: An Analysis Using Grounded Theory. Online Learn. (2018).

[6] Guangzuo, C., Xinqi, R., Haitao, Z., Ronghuai, H.: SMID: A Semantic Model of Instructional Design. In: Proceedings of the 2009 First International Workshop on Education Technology and Computer Science - Volume 03. Pp. 130-134. IEEE Computer Society, Washington, DC, USA (2009). 
[7] Vidal-Castro, C., Sicilia, M.-Á., Prieto, M.: Representing instructional design methods using ontologies and rules. Knowl.-Based Syst. 33, 180-194 (2012).

[8] Göksu, I., Özcan, K.V., Çakir, R., Göktas, Y.: Content Analysis of Research Trends in Instructional Design Models: 1999-2014. J. Learn. Des. 10, 85-109 (2017).

[9] Bolton, D.: Student Attitude and Online Learning: The Impact of Instructional Design Factors upon Attitudes toward the Online Learning Experience. Handb. Res. Humaniz. Distance Learn. Exp. 307-333 (2017).

[10] Lee, J., Jang, S.: A methodological framework for instructional design model development: Critical dimensions and synthesized procedures. Educ. Technol. Res. Dev. 62, 743-765 (2014).

[11] Romiszowski, A.J.: Designing instructional systems: Decision making in course planning and curriculum design. Routledge (2016).

[12] Lee, J., Lim, C., Kim, H.: Development of an instructional design model for flipped learning in higher education. Educ. Technol. Res. Dev. 65, 427-453 (2017).

[13] Londoño Giraldo, E.P.: El diseño instruccional en la educación virtual: más allá de la presentación de contenidos, (2011).

[14] Richey, R. c., Fields, D. c., Foxon, M.: Instructional design competencies: The standards, (2001).

[15] Alvarado, Á.: Instructional Design for the Production of Courses on Line and elearning. IV, 9-24 (2003).

[16] González-Tejero, J.M.S., Parra, R.M.P.: LA CONCEPCIÓN CONSTRUCTIVISTA DE LA INSTRUCCIÓN. RMIE. 13, 32 (2008).

[17] Belloch, C.: Diseño Instruccional. Mater. Didáctico Web Unidad Tecnol. Educ. UTE Univ. Valencia. 21, 2-4 (2013).

[18] Molenda, M.: ADDIE Model DESIGN DEVELOPMENT IMPLEMENTATION. 34-36 (2003).

[19] Esquivel Gámez, I.: Los Modelos Tecno-Educativos, revolucionando el aprendizaje del siglo XXI., México (2014).

[20] Cisneros, B., Eguigure, M., Calderón, S., Pérez, H.: Desarrollo de la comprensión lectora a través de un curso en línea. Un modelo ADDIE. CienciAmérica Rev. Divulg. Científica Univ. Tecnológica Indoamérica. 6, 63-70 (2017).

[21] Mcgriff, S.J.: Instructional System Design (ISD): Using the ADDIE Model. Instr. Syst. Coll. Educ. Penn State Univ. 2 (2000).

[22] Benitez Lima, M.G.: El modelo de diseño instruccional ASSURE aplicado a la educación a distancia. Tlatemoani1 Recuperado Httpwwweumednetrevtlatemoanio1mgblhtm. 13 (2010). 
[23] Dávila, A.A., Pérez, J.F.: Diseño instruccional de la educación en línea usando el modelo ASSURE. EDUCARE. 0, (2007).

[24] Leoni, S.: METODOLOGÍA PACIE: UN CAMBIO REVOLUCIONARIO EN LA EDUCACIÓN VIRTUAL. Cuad. Educ. YDesarrollo. 2, (2010).

[25] Flores, K.M., Bravo, M. de la S.: Metodología Pacie En Los Ambientes Virtuales De Aprendizaje Para El Logro De Un Aprendizaje Colaborativo. Diálogos Educ. 12, 3-17 (2013). 